\title{
Embolization Using Endovascular Technique in Acute and Chronic Stages of Traumatic Ophthalmic Artery Aneurysm -Case Report-
}

\author{
Ryuzaburo KANAZAWA, ${ }^{1}$ Shoichiro ISHIHARA, ${ }^{1}$ Hiroaki NEKI, ${ }^{1}$ Mai OKAWARA, ${ }^{1}$ \\ Hideaki ISHIHARA, ${ }^{1}$ Shinya KOHYAMA, ${ }^{1}$ Fumitaka YAMANE, ${ }^{1}$ and Akira SATO ${ }^{2}$
}

Divisions of ${ }^{1}$ Endovascular Neurosurgery and ${ }^{2}$ Surgical Neurosurgery, Stroke Center, Internal Medical Center, Saitama Medical University, Hidaka, Saitama

\begin{abstract}
A 44-year-old man presented with traumatic aneurysm of the left ophthalmic artery. The first coil embolization intervention achieved relatively tight packing of the aneurysm and the parent artery. After 5 months, a second embolization procedure was required because of recurrence of the aneurysm with transition of the intraaneurysmal coil formation. No recurrence occurred after the second embolization. We thought that the first embolization might have prevented catastrophic rupture, whereas the second embolization resulted in complete obliteration of the aneurysm. No entity of the aneurysmal wall formation of the current traumatic aneurysm in the acute stage may have resulted in the recanalization of the aneurysm followed by the second intervention. The stability of the wall in the chronic stage may correlate with the complete obliteration.
\end{abstract}

Key words: traumatic aneurysm, ophthalmic artery, endovascular treatment, follow up, blunt trauma

\section{Introduction}

Traumatic aneurysm is a dangerous lesion that should be treated as swiftly as possible. However, the aneurysm wall consists of surrounding connective tissue, and so may prove fragile during treatment. Traumatic ophthalmic artery aneurysm is very rare and difficult to completely obliterate because of the anatomical location, and the extensive anastomoses with the facial and ethmoidal branches of the external carotid arteries (ECAs). ${ }^{7}$ We report a case of traumatic ophthalmic artery aneurysm that required repeat endovascular embolizations in the acute and chronic stages.

\section{Case Report}

A healthy 44-year-old man was referred to our hospital after falling from a dump truck at work. On arrival, he was free of neurological symptoms with Glasgow Coma Scale score of 15. Initial computed tomography (CT) demonstrated left sylvian cistern traumatic subarachnoid hemorrhage (SAH) without focal concentration of $\mathrm{SAH}$ in the supraclinoid left internal carotid artery (ICA) region.

The patient complained of gradually progressive disturbance of visual acuity in his left eye and was transferred to our department after consultation. On admission, he was blind in the left eye with a dilated pupil. Radiological ex-

Received January 8, 2010; Accepted August 17, 2010 amination disclosed no compression of the left ophthalmic nerve caused by fractures of the optic canal. Therefore, craniotomy for decompressive surgery was not considered and conservative treatment with steroids was administered. No direct injury to the eyeball was identified but ophthalmologic examination revealed ischemic transformation of the fundus. We speculated that distal embolism had migrated to the retinal artery in the process of the aneurysm formation and caused this visual disturbance.

On the 6th day after admission, follow-up three-dimensional (3D)-CT angiography revealed an aneurysm of the left intracranial dorsal ICA (Figs. 1 and 2). Urgent angiography revealed an aneurysm of the ophthalmic artery with no significant findings in the anterior genu and the supraclinoid segments of the left ICA (Fig. 3A). We suspected that the aneurysm was a false aneurysm with the possibility of rupture. Endovascular embolization was chosen as the treatment with the patient's informed consent. During the entire clinical course, the patient's neurological findings were normal except for the visual disturbance. Cross-flow circulation through the anterior and posterior communicating arteries was relatively poor, which suggested intolerance of left ICA occlusion. Therefore, no balloon occlusion test was performed.

The embolization procedure was performed under general anesthesia. A 7F guiding catheter (Britetip; Cordis Endovascular Systems, Miami Lakes, Florida, USA) was introduced through the left ICA. A Hyperform balloon 


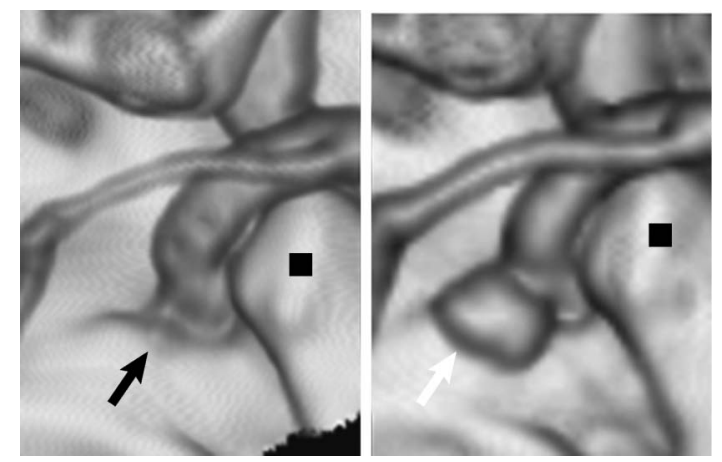

Fig. 1 Detection of traumatic aneurysm formation. Left: Three-dimensional (3D)-computed tomography (CT) angiogram on admission on the day after the injury showing no aneurysm formation (arrow). The patient had already suffered progressive visual disturbance of the left eye. Right: Follow-up 3D-CT angiogram 6 days after the first study showing an aneurysm (arrow). Black squares: left anterior clinoid process.
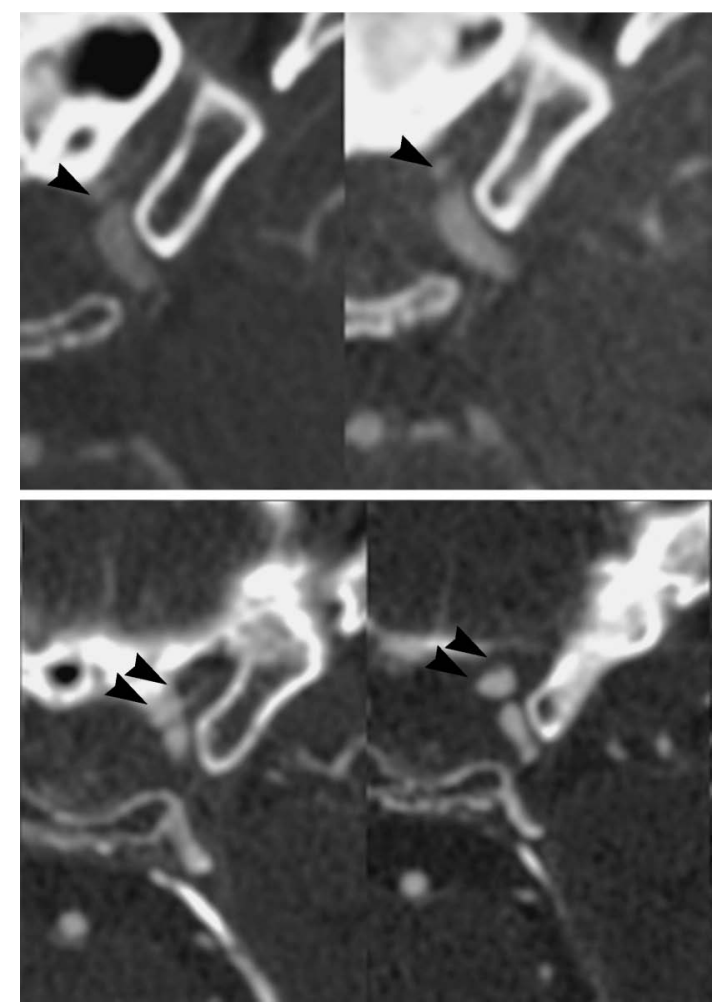

Fig. 2 Detection of formation of traumatic aneurysm. Upper row: Three-dimensional (3D)-computed tomography (CT) angiograms on admission on the day after the injury showing no aneurysm formation or thrombosed aneurysm, and the proximal part of the ophthalmic artery close to the wall of the optic canal (arrowheads). Collision between the proximal artery and the wall may be connected with the formation of the traumatic aneurysm. Lower row: Follow-up 3D-CT angiograms 6 days later showing the aneurysm (double arrowheads). catheter (eV3 Neurovascular, Irvine, California, USA) was placed in the origin of the ophthalmic artery for prevention of coil migration into the ICA, followed by insertion of a microcatheter (SL-10; Boston Scientific, Natick, Massachusetts, USA) into the aneurysm. An extra soft $2.5-\mathrm{mm}$ helix $\times 6$-cm long ED coil (Kaneka, Osaka) was inserted through the microcatheter into the aneurysm for prevention of injury to the fragile wall of the aneurysm. After that, seven additional ED coils were inserted resulting in complete obliteration of the aneurysm (Fig. 3B). The goal of the procedure was to prevent rupture of the aneurysm and the associated irreversible visual loss. To ensure embolization, we attempted to occlude the vessel from the neck of the aneurysm to the origin of the ophthalmic artery. ED coils were employed as the embolic material because of the possibility that the aneurysm wall consisted of surrounding connective tissues and hematoma. No complication occurred in the postoperative period. Follow-up angiography performed one week later excluded recurrence of the aneurysm. The patient was discharged on the 11th postoperative day with a blind left eye.

Follow-up angiography scheduled 3 months after the first operation revealed partial recanalization with compaction of the intraaneurysmal coil formation (Fig. 3C). Surveillance angiography 2 months later revealed progressive filling of the intraaneurysm dome (Fig. 3D). After lengthy discussion with the patient, re-treatment was planned with informed consent. The second operation was performed under general anesthesia. We achieved complete obliteration of the aneurysm with an additional 2 -mm helix $\times 6$-cm long ED coil (Fig. 3E). No recanalization was observed for a year after the second operation (Fig. 3F).

\section{Discussion}

The present unique case of traumatic ophthalmic artery aneurysm occurred within several days of a nonpenetrating injury in a patient who presented with SAH. The aneurysm was identified at the second CT angiography and was successfully treated with two coil embolization procedures. The initial CT findings suggested that the hemorrhage resulted from trauma and not from rupture of a previously existing aneurysm. 3D-CT angiography had detected no signs of thrombosed aneurysm on arrival (Figs. 1 and 2). Most cases of traumatic aneurysm do not have any neck, and the walls are formed of surrounding connective tissues or hematoma without true vessel wall components. ${ }^{9)}$ Therefore, we thought that the aneurysm was caused by external trauma, and the lesion was most likely to be a false aneurysm.

The goal of treatment of traumatic aneurysms is to prevent life-threatening hemorrhage by excluding the aneurysm from the circulation by surgical or endovascular procedures. Some surgical treatment options for ophthalmic artery lesions, such as proximal clipping of the ophthalmic artery originating from the ICA ${ }^{1,2,12)}$ and ICA ligation $^{8)}$ have been reported. The extraorbital ophthalmic artery branches have extensive anastomoses with the facial and ethmoidal branches of the ECAs. ${ }^{7}$ Therefore, 

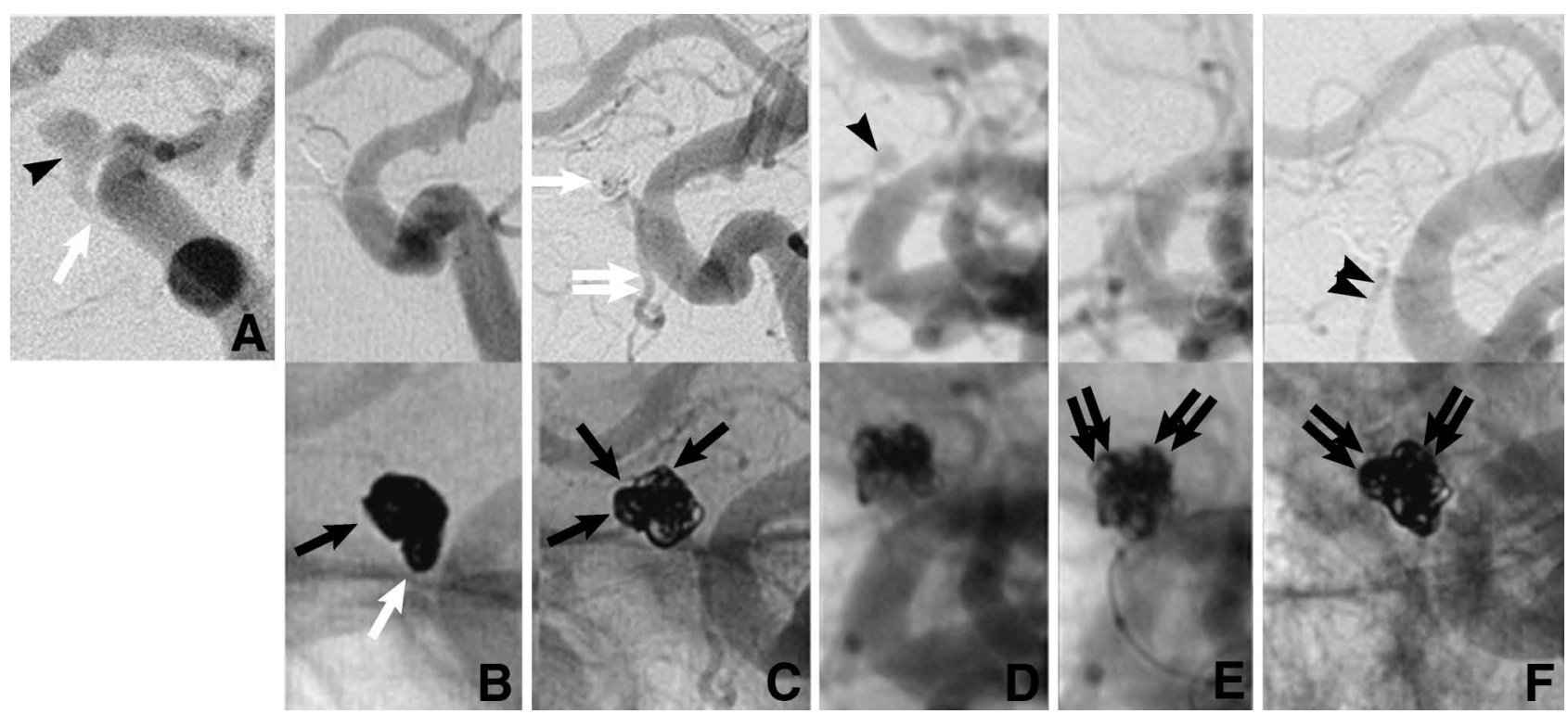

Fig. 3 Coil embolization, surveillance angiography, and transition of coil formation. Upper row: Digital subtraction angiograms, lower row: corresponding angiograms at each time point in the upper row. A: Preoperative angiogram showing the aneurysm with a broad neck, located at the hook region of the ophthalmic artery (arrowhead), not at the bifurcation between the internal carotid artery and the ophthalmic artery (arrow). B: Angiograms of the final stage of the first embolization showing relatively tight packing of the coil mass with an intraaneurysmal compartment (black arrow) and intraophthalmic artery compartment (white arrow). C: Angiograms taken 3 months after the operation showing coil compaction (white arrow) and recanalization of the ophthalmic artery (double white arrows). Coil compaction and the change of intraaneurysmal coil formation (black arrows) was due to the fragile aneurysm wall yielding to the elasticity of the coil. Conservative treatment was begun. D, E: Angiograms taken 5 months after the first procedure (D) and the second operation (E) showing progressive partial intraaneurysm filling (arrowhead), followed by successful second embolization. The compaction progressed while the intraaneurysmal coil formation seemed relatively stable (double black arrows), suggesting maturity of the surrounding fibrous wall. F: Angiograms taken 3 months after the second operation showing no signs of recurrence, and the entire coil formation appeared to be stable (double black arrows). The ophthalmic artery flow was maintained (double arrowheads).

complete obliteration of the aneurysm at its neck is difficult by proximal occlusion. ${ }^{10)}$ Moreover, neck clipping or trapping of both sides of the aneurysm may not be feasible because of the fragile nature and location of the aneurysm. ${ }^{10)}$ In the present case, endovascular treatment was preferred over conventional surgical procedures to guarantee the isolation of the aneurysm from the circulatory system. However, obliteration of an aneurysm with maintenance of the parent artery flow is not always appropriate and safe because the wall of the aneurysm may be formed by thickened arachnoid and surrounding cortex in the acute stage without development of a neck. ${ }^{3}$ Therefore, endovascular occlusion of the parent vessel may be more suitable for safer and guaranteed treatment. ${ }^{111}$

Active and rapid treatment of such lesions is necessary because rupture usually occurs within 2-3 weeks after trauma. ${ }^{3,10)}$ In the present case, the fragile nature of the aneurysm wall was the main cause of regrowth of the aneurysm. The true aneurysm wall supporting the stable coil basket against the blood pressure may be absent in the acute stage of traumatic false aneurysm. We considered that collision between the proximal part of the ophthalmic artery and the optic canal wall resulted in the occurrence of the aneurysm in the current case. The nonrecurrence after retreatment in the chronic stage may be due to the presence of matured "false" aneurysmal wall. Ten cases of traumatic aneurysm originating from the intracranial ophthalmic artery have been reported. ${ }^{1,2,4-8,13,14)}$ Only two cases were treated with endovascular technique, but the long-term follow up of the patients was not reported.7,14) All reported cases of acute traumatic ophthalmic artery aneurysms treated with endovascular embolization in the acute stage were cured by aneurysm obliteration and occlusion of the parent ophthalmic artery. ${ }^{7}{ }^{14)}$ Embolization in the acute stage, including that of the parent artery, may guard against direct pressure to the fragile aneurysm wall. Treatment of the pseudoaneurysm of the parent vessel may be fraught with complications owing to the fragility of the wall of the pseudoaneurysm. ${ }^{11)}$ Over a period of weeks, a fibrous wall matures around the pseudoaneurysm, which may allow direct coil embolization with careful microcatheter manipulation. ${ }^{7)}$ The only case of successful neck clipping of bilateral ophthalmic artery pseudoaneurysms treated 3 months after injury was suggestive of this proposed process. ${ }^{6)}$

Endovascular strategy for traumatic ophthalmic artery aneurysm was useful for achieving complete obliteration of the aneurysm in the present case. However, this lesion may not have a true wall, only surrounding connective tissue or hematoma, so normal embolization of the aneurysm 
to retain the patency of ophthalmic artery may not be feasible, and simultaneous parent artery occlusion may be inevitable. Simultaneous aneurysm embolization may prevent retrograde flow from the distal site of the ophthalmic artery to the aneurysm, so may have an advantage over parent artery proximal clipping. However, balloon test occlusion of the ICA and concurrent evaluation of the collateral flow to the ophthalmic artery before treatment should be considered. Pure intraaneurysmal embolization under close follow up or alternative treatment should be considered for intolerant patients.

We employed soft ED coils for gentle insertion and to minimize stress to the wall. To prevent catastrophic rupture of such aneurysms, surveillance follow-up angiography may be recommended until the chronic stage. Long-term follow up of the aneurysm may ensure permanent obliteration of the aneurysm. Further case studies and development of endovascular materials and technology may be necessary to improve the treatment of these delicate and fragile lesions.

\section{References}

1) Aarabi B: Management of traumatic aneurysms caused by high-velocity missile head wounds. Neurosurg Clin N Am 6: 775-797, 1995

2) Amirjamshidi A, Abbassioun $K$, Rahmat $H$ : Traumatic aneurysms and arteriovenous fistulas of the extracranial vessels in war injuries. Surg Neurol 53: 136-145, 2000

3) Bousquet C, Lejeune JP, Christiaens JL: Traumatic aneurysm of the supraclinoid internal carotid artery. Case report. Surg Neurol 31: 319-322, 1989

4) Fleischer AS, Patton JM, Tindall G: Cerebral aneurysms of traumatic origin. Surg Neurol 4: 233-239, 1975

5) Haddad FS, Haddad GF, Taha J: Traumatic intracranial aneurysms caused by missiles: their presentation and management. Neurosurgery 28: 1-7, 1991

6) Hahn YS, McLone DG: Traumatic bilateral ophthalmic ar- tery aneurysms: a case report. Neurosurgery 21: 86-89, 1987

7) Hopkins JK, Shaibani A, Ali S, Khawar S, Parkinson R, Futterer S, Russell EJ, Getch C: Coil embolization of posttraumatic pseudoaneurysm of the ophthalmic artery causing subarachnoid hemorrhage. Case report. J Neurosurg 107: 1043-1046, 2007

8) Kinley GJ, Leighinger DS: Aneurysm of anomalous ophthalmic artery presenting in the sphenoid sinus and simulating an aneurysm of the internal carotid artery on routine arteriography. J Neurosurg 9: 544-547, 1952

9) Kubota S: [No Kekkan Shogai Wo Kiwameru]. Osaka, Nagai Shoten, 2004, pp 90-96 (Japanese)

10) Lee CY, Yim MB, Kim IM, Son EI, Kim DW: Traumatic aneurysm of the supraclinoid internal carotid artery and an associated carotid-cavernous fistula: vascular reconstruction performed using intravascular implantation of stents and coils. Case report. J Neurosurg 100: 115-119, 2004

11) Lempert TE, Halbach VV, Higashida RT, Dowd CF, Urwin RW, Balousek PA, Hieshima GB: Endovascular treatment of pseudoaneurysms with electrolytically detachable coils. AJNR Am J Neuroradiol 19: 907-911, 1998

12) Rahmat $H$, Abbassioun $K$, Amirjamshidi A: Pulsating unilateral exophthalmos due to traumatic aneurysm of the intraorbital ophthalmic artery. Case report. J Neurosurg 60: 630-632, 1984

13) Tantana S, Pilla TJ, Awwad EE, Smith KR: Balloon embolization of a traumatic carotid-ophthalmic pseudoaneurysm with control of the epistaxis and preservation of the internal carotid artery. AJNR Am J Neuroradiol 8: 923-924, 1987

14) Vora NA, Germanwala AV, Thomas AJ, Horowitz MB, Jovin TG: Endovascular management of a traumatic ophthalmic artery aneurysm. J Trauma 63: 192-194, 2007

Address reprint requests to: Ryuzaburo Kanazawa, MD, PhD, Division of Endovascular Neurosurgery, Stroke Center, Internal Medical Center, Saitama Medical University, 1397-1 Yamane, Hidaka, Saitama 350-1298, Japan. e-mail: ryu.kan.jp.2412@gmail.com 